\title{
MELD score and serum sodium in the prediction of survival of patients with cirrhosis awaiting liver transplantation
}

\author{
Maria-Carlota Londoño, Andrés Cárdenas, Mónica Guevara, Llorenç Quintó, Dara de las Heras, \\ Miguel Navasa, Antoni Rimola, Juan-Carlos Garcia-Valdecasas, Vicente Arroyo, Pere Ginès
}

See end of article for authors' affiliations

Correspondence to: Pere Ginès, MD, Liver Unit, Hospital Clínic de Barcelona, C/Villarroel 170, 08036, Barcelona, Spain; pgines@ clinic.ub.es

Revised 5 April 2007 Accepted 10 April 2007 Published Online First 23 April 2007

\begin{abstract}
Background/Aims: Serum sodium predicts prognosis in cirrhosis and may improve the prognostic accuracy of the model for end-stage liver disease (MELD) score, but the available information is limited. The aim of the present study was to assess the prognostic value of serum sodium in the prediction of survival at 3 and 12 months after listing in patients with cirrhosis awaiting liver transplantation, and to compare its predictive value with that of the MELD score.

Patients and methods: 308 consecutive patients with cirrhosis listed for transplantation during a 5-year period were included in the study. The end-point was survival at 3 and 12 months before transplantation. Variables obtained at the time of listing were analysed for prognostic value using multivariable analysis. Accuracy of prognostic variables was analysed by receiver operating characteristic (ROC) curves.

Results: The MELD score and serum sodium concentration were the only independent predictors of survival at 3 and 12 months after listing. Low serum sodium was associated with an increased risk of death in all subpopulations of patients with cirrhosis categorised according to the major complication developed before listing. The area under the ROC curves for serum sodium and MELD score was not significantly different both at 3 months $(0.83$ vs 0.79 , respectively) and at 12 months ( 0.70 vs 0.77 , respectively). The addition of serum sodium did not significantly improve the accuracy of the MELD score in the prediction of survival at 3 and 12 months.

Conclusion: In patients with cirrhosis awaiting liver transplantation, serum sodium and MELD were found to be independent predictors of survival. Larger studies are needed to determine whether the addition of serum sodium to MELD can improve its prognostic accuracy.
\end{abstract}

$\mathrm{T}$ he model for end-stage liver disease (MELD) score is the method most widely used for organ allocation in liver transplantation. ${ }^{1-3}$ This model, which includes variables related to both liver and renal function, was implemented in the USA in 2002 and is currently being used in many countries to classify patients with cirrhosis awaiting transplantation according to the severity of their liver disease. Nevertheless, several studies, as well as clinical observation, indicate that some subsets of patients with cirrhosis may have high mortality despite low MELD scores. ${ }^{4-7}$ Therefore, there is need for improvement of the MELD score. In this regard, several recent studies have shown that serum sodium concentration is a good marker of prognosis in patients awaiting transplantation. ${ }^{8-10}$ According to the results of these studies, the use of serum sodium in the assessment of severity of cirrhosis has been recommended. However, there are several issues on the possible use of serum sodium as a predictor of prognosis that require more information, specifically: (1) whether the value of serum sodium is equally effective in the assessment of short-term prognosis (3 months) compared with mid-term prognosis (12 months); (2) whether serum sodium is equally accurate in predicting prognosis in different subpopulations of patients with cirrhosis; and (3) whether serum sodium improves the accuracy of the MELD score. The purpose of this study was to investigate these issues.

\section{PATIENTS AND METHODS \\ Study population}

This is a single-centre retrospective cohort study of all adult patients with cirrhosis listed for liver transplantation at the Hospital Clínic of Barcelona during the period between January 2000 and March 2005. During this period, a total of 560 patients were listed for transplantation. Two hundred and fifty-two patients were excluded from this study for the following reasons: hepatocellular carcinoma $(n=152)$, retransplantation $(\mathrm{n}=48)$ or diseases other than cirrhosis (acute liver failure, $\mathrm{n}=22$; familial amyloidotic polyneuropathy, $\mathrm{n}=18$; or miscellaneous disorders, $n=12$ ). Patients in whom hepatocellular carcinoma was diagnosed after listing $(n=2)$ and those who were found to have incidental hepatocellular carcinoma at transplantation $(n=12)$ were not excluded from the study. The study cohort included 308 patients with cirrhosis.

Criteria for listing patients with cirrhosis for liver transplantation throughout the study period were the existence of decompensated liver disease together with moderate-to-severe liver failure, as indicated by a Child-Pugh score $\geqslant 7$, and absence of absolute contraindications for transplantation. Patients belonging to Child-Pugh class A were only considered for transplantation if they had developed hepatic encephalopathy or had a concomitant parenchymal renal disease requiring combined liver-kidney transplantation. ${ }^{11}{ }^{12}$ At the time of listing, demographic, clinical and biochemical variables were collected and included in a specific database for patients awaiting transplantation.

Once included on the waiting list, patients were followed-up by experienced hepatologists, and complications of cirrhosis were treated according to standardised therapeutic protocols as follows. Moderate ascites was treated with low-sodium diet and diuretics (spironolactone alone or in combination with furosemide), and large ascites was treated with large-volume paracentesis plus albumin, followed by low-sodium diet and

Abbreviations: INR, international normalisation ratio; MELD, model for end-stage liver disease; ROC, receiver operating characteristic 
diuretics, as described in detail elsewhere. ${ }^{13}{ }^{14}$ Ascites at the time of listing was classified as controlled or uncontrolled according to the score obtained in the Child-Pugh classification, 2 or 3 , respectively. ${ }^{15}{ }^{16}$ Spontaneous bacterial peritonitis was treated with ceftriaxone plus intravenous albumin, followed by the administration of norfloxacin to prevent recurrence. ${ }^{14}{ }^{17}$ Dilutional hyponatraemia (serum sodium $<130 \mathrm{mEq} / \mathrm{l}$ ) was managed with fluid restriction (1-1.5 l/ day), except for those patients with severe hyponatraemia ( serum sodium $<120 \mathrm{mEq} / \mathrm{l}$ ), who were given hypertonic saline before transplantation in an attempt to increase serum sodium concentration. Hepatorenal syndrome with serum creatinine above $2 \mathrm{mg} / \mathrm{dl}$ was managed with terlipressin plus albumin. ${ }^{18}$ Acute variceal bleeding was initially managed with either somatostatin or terlipressin associated with emergency sclerotherapy or band ligation. Prevention of recurrent bleeding was performed with the administration of $\beta$-blockers with or without variceal band ligation. ${ }^{19} 20$ Acute hepatic encephalopathy was treated with lactulose and rectal enemas, and treatment of the precipitating cause, if any. ${ }^{17}$

During the study period (January 2000 to March 2005), the allocation of livers for transplantation in our centre was strictly determined by the time on the waiting list; in other words, when a liver from a cadaveric donor became available, the liver was assigned to the patient who had been on the waiting list for the longest time, matched by $\mathrm{ABO}$ group and body weight. Living-related liver transplantation was offered to suitable candidates. Since April 2005, the allocation of livers in our transplant programme is based on the severity of liver disease using the MELD score.

\section{End-points and definitions}

The primary end-points of the study were survival before transplantation at 3 and 12 months after inclusion on the waiting list. The MELD score at inclusion was calculated according to the formula of the United Network for Organ Sharing (UNOS) available at www.unos.org. Hyponatraemia was defined as serum sodium $<130 \mathrm{mEq} / \mathrm{l}$ at the time of listing, according to the definition of the International Ascites Club. ${ }^{21}$ Renal failure was defined as the presence of serum creatinine $\geqslant 1.5 \mathrm{mg} / \mathrm{dl}$ at the time of listing. Hepatorenal syndrome was diagnosed using the criteria of the International Ascites Club, as reported elsewhere. ${ }^{22}$

\section{Statistical analysis}

Demographic, clinical and biochemical variables were analysed as possible predictors of survival in a univariate analysis, and probability curves (Kaplan-Meier) were compared with the logrank test. Values of biochemical variables included in the statistical analysis were concurrent and obtained at the time of inclusion of patients on the waiting list. Of all clinical and biochemical variables analysed in the whole series of patients, there were only a few missing values (bilirubin in 6 patients, albumin in 6, international normalisation ratio (INR) in 5, serum creatinine in 1 , and MELD score in 9). Missing values were considered as missing for the statistical analysis and were not replaced by any values. Two different survival analyses were performed: at 3 and 12 months after inclusion on the waiting list. The 3-month survival analysis included all deaths occurring between day 0 and day 90, while the 12-month survival analysis included all deaths between day 0 and day 365 . Transplanted patients were considered as censored at the time of transplantation. Twenty-four patients were censored for transplantation at 3 months, and 174 patients were censored for transplantation at 12 months. Patients removed from the waiting list because of being "too sick" to be transplanted were considered as dead at the time of exclusion from the waiting list. Patients removed from the waiting list because of improvement of their liver function and those removed because of listing in another transplant centre were considered as censored at the time of exclusion from the waiting list. Patients still alive on the waiting list at the end of each period ( 3 and 12 months) were considered as censored. Predictive factors identified in the univariate analysis were included in the multivariable analysis, using Cox regression method. A stepwise forward regression method was used. Validation of the proportional hazards assumption for the final models (ie, that the relative risk of failure between subgroups in the model does not change over time) was carried out through a graphical examination of log minus log plots of the Kaplan-Meier survival curves versus the log of time, for the tertiles of the model prediction. ${ }^{23}{ }^{24}$ The final models were validated using the bootstrap method. ${ }^{25} 26$

The accuracy of each independent predictive factor of survival was assessed by receiver operating characteristic (ROC) curves. Comparison between ROC curves (concordance c-statistic test) was performed with the statistical package available at www.analyse-it.com, which uses the algorithm described by Hanley and McNeil. ${ }^{27}$ Statistical analysis was performed using SPSS 10 for Windows (SPSS Inc., Chicago, IL, USA). Results are expressed as mean (SD). $\mathrm{p}<0.05$ was considered as statistically significant. The study was approved by the Institutional Review Board of the Hospital Clínic of Barcelona.

\section{RESULTS}

\section{Characteristics of the patients}

Demographic, clinical and biochemical data of patients included in the study at the time of listing are shown in table 1 . One hundred and thirty-five (44\%) of the 308 patients had an abnormal serum sodium concentration $(<135 \mathrm{mEq} / \mathrm{l})$. The prevalence of low serum sodium concentration as defined by a serum sodium concentration $<130,<125$ and $<120 \mathrm{mEq} / \mathrm{l}$ was 14,5 and $1 \%$, respectively.

\section{Survival of patients on the waiting list}

One-hundred and ninety (62\%) out of the 308 patients included in the study underwent liver transplantation during follow-up (157 patients from deceased donors, 22 patients from living donors and 11 patients received a domino liver transplantation), 65 patients $(21 \%)$ died while in the waiting list, 18 patients $(6 \%)$ were removed because of listing in another transplant centre, 7 patients $(2 \%)$ were excluded from the list either because they were too sick to be transplanted ( 4 patients) or because of marked improvement of liver function (3 patients), and 28 patients $(9 \%)$ were still awaiting transplantation at the time of the analysis of the results. The median time on the waiting list was 6.4 months (range $0.1-$ 18 months). Figure 1 shows the probability of survival on the waiting list of the whole population of included patients. The probability of survival before transplantation was $88 \%$ at 3 months and $68 \%$ at 12 months after inclusion on the waiting list.

\section{Predictive factors of survival on the waiting list}

The predictive factors of survival were analysed at two different time points: 3 and 12 months.

\section{Three-month survival}

Thirty-four $(11 \%)$ of the 308 patients died within the first 3 months after inclusion on the waiting list before transplantation was performed. Table 2 shows the comparison of clinical and biochemical characteristics of patients who died and those of patients who survived the initial 3 months. Factors associated with 3-month survival in the univariate analysis 
Table 1 Demographic and clinical data and liver and renal function tests at the time of listing for transplantation in the 308 patients included in the study

\begin{tabular}{|c|c|}
\hline Age (years) & $52(9)(18-66)$ \\
\hline \multicolumn{2}{|l|}{ Sex } \\
\hline Male & 207 (67\%) \\
\hline Female & $101(33 \%)$ \\
\hline \multicolumn{2}{|l|}{ Aetiology of cirrhosis } \\
\hline Hepatitis C & $136(44 \%)$ \\
\hline Alcohol & $80(26 \%)$ \\
\hline Hepatitis $C$ and alcohol & $25(8 \%)$ \\
\hline Hepatitis B & $20(6 \%)$ \\
\hline Other* & 47 (18\%) \\
\hline \multicolumn{2}{|l|}{ Complications of cirrhosis } \\
\hline Ascites & $271(88 \%)$ \\
\hline Controlled & $112(41 \%)$ \\
\hline Uncontrolled & $159(59 \%)$ \\
\hline Hepatic encephalopathy & $145(47 \%)$ \\
\hline Variceal bleeding & $91(29 \%)$ \\
\hline SBP & $83(27 \%)$ \\
\hline Renal failure $†$ & 39 (13\%) \\
\hline Hepatorenal syndromeł & $20(7 \%)$ \\
\hline Parenchymal renal disease & $19(6 \%)$ \\
\hline Bilirubin $(m g / d l) \S$ & $4.3(5)(0.2-46)$ \\
\hline Albumin (g/l) & $30(5)(17-45)$ \\
\hline \multicolumn{2}{|l|}{ Prothrombin time } \\
\hline Ratio & $54(16)(14-100)$ \\
\hline INR & $1.5(0.8)(1-12)$ \\
\hline Creatinine $(\mathrm{mg} / \mathrm{dll}) \boldsymbol{\sigma}$ & $1.2(0.9)(0.3-11)$ \\
\hline Sodium (mEq/l) & $135(5)(111-146)$ \\
\hline MELD score & $18(5)(7-48)$ \\
\hline Child-Pugh score & $9.3(1.8)(5-15)$ \\
\hline \multicolumn{2}{|c|}{ 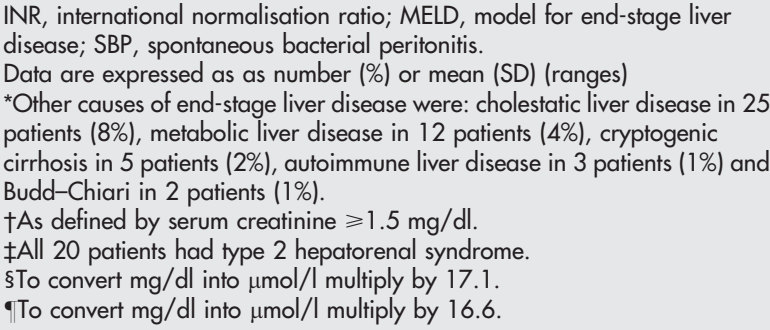 } \\
\hline
\end{tabular}

were: hepatorenal syndrome, uncontrolled ascites, serum bilirubin, serum albumin, INR, serum sodium concentration and MELD score. All these variables were included in the multivariable analysis as they were considered clinically relevant. In multivariable analysis, only serum sodium and MELD score were independently associated with prognosis (table 3). When the MELD score was excluded from the multivariable analysis, factors that were independently associated with prognosis were: serum bilirubin, serum creatinine and serum sodium concentration. Figure 2 shows the individual relationship between serum sodium and MELD score and 3month probability of survival. There was a $12 \%$ increase in the risk of death for each unit $(\mathrm{mEq} / \mathrm{l})$ decrease in serum sodium concentration between 120 and $135 \mathrm{mEq} / \mathrm{l}$. Similarly, for each unit of increase in MELD score (between 15 and 40), the risk of death increased by $8 \%$. The area under the ROC curves for MELD score and serum sodium were 0.79 (95\% CI 0.71-0.86) and 0.83 (95\% CI $0.76-0.90)$, respectively, the difference not being statistically significant $(\mathrm{p}=0.4)$.

\section{Twelve-month survival}

Sixty-four $(21 \%)$ of the 308 patients died within the first 12 months after inclusion on the waiting list before transplantation was performed. Table 4 shows the comparison of clinical and biochemical characteristics of patients who died and those of patients who survived within the initial 12-month period. Factors associated with 12-month survival in the univariate analysis were: hepatic encephalopathy, hepatorenal syndrome,

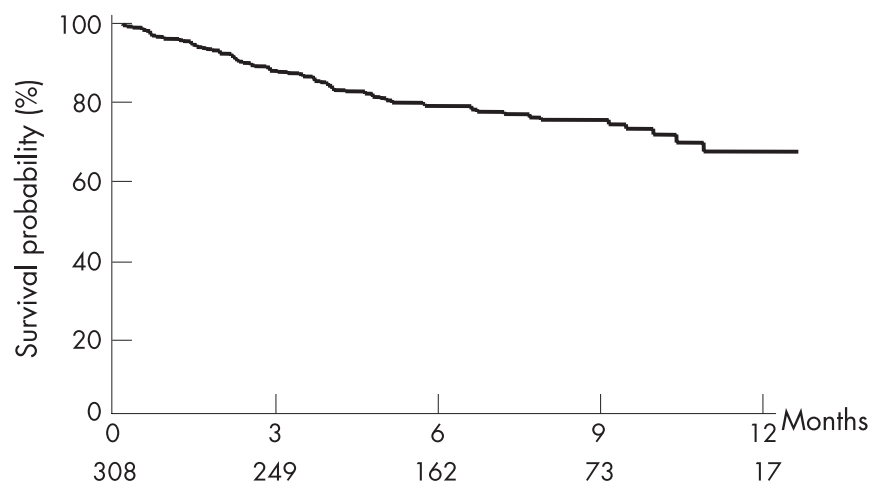

Figure 1 One-year survival before transplantation of the whole population of patients with cirrhosis included in the study. Time 0 is the time of listing. Numbers under the graph are patients at risk at each time point.

uncontrolled ascites, renal failure, serum bilirubin, INR, serum albumin, serum sodium concentration and MELD score. All these variables were included in the multivariable analysis as they were considered clinically relevant. In multivariable analysis, again serum sodium and MELD score were independently associated with prognosis (table 3). MELD score at listing in patients transplanted during the 12 -month period $(\mathrm{n}=190)$ was 15.9 (4.2) compared with 21 (5) in patients who died during the same period $(n=64)$. Corresponding values of serum sodium at listing were 136 (4) vs 132 (6) mEq/l, respectively $(\mathrm{p}<0.05$ for both). When the MELD score was excluded from the multivariable analysis, factors that were independently associated with prognosis were: serum bilirubin, serum creatinine, INR and serum sodium concentration. Figure 3 shows the probability of survival during the first year after inclusion on the waiting list for all patients classified according to different values of serum sodium and MELD score. The area under the ROC curves for MELD score and serum sodium were 0.77 (95\% CI 0.70 to 0.80 ) and 0.70 (95\% CI 0.60 to 0.78$)$, respectively, the difference not being statistically significant $(\mathrm{p}=0.14)$.

To assess the possible value of serum sodium in improving the accuracy of the MELD score in the evaluation of prognosis, serum sodium concentration was incorporated in the calculation of the MELD score, using a formula described recently. ${ }^{28} \mathrm{~A}$ MELD-Na score was then calculated for each patient and its accuracy in predicting prognosis was compared with that of the MELD score and serum sodium using ROC curves. As shown in fig 4, no significant differences were observed between the predictive value of MELD-Na at 3 and 12 months and those of the MELD score and serum sodium concentration calculated individually. The addition of the variable uncontrolled ascites to MELD-Na did not significantly improve the accuracy of MELD alone or MELD-Na in the prediction of 3- or 12-month survival (area under the ROC curve for 3-month survival: MELD 0.79, MELD-Na 0.83, MELD-Na-uncontrolled ascites 0.84; area under the ROC curve for 12-month survival: MELD 0.76; MELD-Na 0.78, MELD-Na-uncontrolled ascites 0.77). Moreover, when a separate analysis was performed in the subgroup of patients with low MELD $(<21),{ }^{8}$ no significant differences were found between the predictive values of MELD-Na and MELD-Na-uncontrolled ascites and those of MELD score or serum sodium individually (area under the ROC curve for 3-month survival: MELD 0.74, serum sodium 0.79, MELD-Na 0.80, MELD-Na-uncontrolled ascites 0.81; area under the ROC curve for 12-month survival: MELD 0.70, serum sodium 0.62, MELD-Na 0.70, MELD-Na-uncontrolled ascites $0.60)$. 
Table 2 Comparison of clinical and biochemical characteristics of patients who died and those of patients who survived the initial 3-month period

\begin{tabular}{lccll}
\hline & Dead $(\mathbf{n}=\mathbf{3 4})$ & Alive $(\mathbf{n = 2 7 4 )}$ & $\mathrm{p}$ Value & Likelihood ratio \\
\hline Age (years) & $53(8)$ & $52(9)$ & 0.37 & \\
Ascites & $33(97 \%)$ & $238(87 \%)$ & & \\
$\quad$ Controlled & $6(18 \%)$ & $106(44 \%)$ & 0.004 & 7.25 \\
$\quad$ Uncontrolled & $27(82 \%)$ & $132(56 \%)$ & & \\
Hepatic encephalopathy & $18(53 \%)$ & $127(46 \%)$ & 0.58 & 0.49 \\
GI bleeding & $10(29 \%)$ & $81(29 \%)$ & 0.57 & 0.001 \\
SBP & $12(35 \%)$ & $71(26 \%)$ & 0.37 & 0.77 \\
Renal failure* & $6(17 \%)$ & $33(12 \%)$ & 0.48 & 5.21 \\
Hepatorenal syndrome† & $6(17 \%)$ & $14(5 \%)$ & 0.026 & \\
Bilirubin (mg/dl) & $8.5(8.4)$ & $3.6(3.1)$ & 0.000 & \\
Albumin (g/l) & $28(5)$ & $31(5)$ & 0.000 & \\
INR & $1.8(0.5)$ & $1.6(0.9)$ & 0.025 & \\
Creatinine (mg/dl) & $1(0.3)$ & $1.2(1.1)$ & 0.416 & \\
Sodium (mEq/l) & $130(6)$ & $136(4)$ & 0.000 & \\
MELD score & $21(5)$ & $16(5)$ & 0.000 & \\
\hline Gl, gastrointestinal; INR, international normalisation ratio; MELD, model for end-stage liver disease. \\
*As defined by serum creatinine $\geqslant 1.5$ mg/dl. \\
TAll patients had type 2 hepatorenal syndrome.
\end{tabular}

Serum sodium and survival in different subpopulations of patients with cirrhosis

To assess further the prognostic value of serum sodium concentration, patients were categorised according to the major complication(s) of cirrhosis they had developed before listing. The prognostic value of serum sodium was then evaluated in these subsets of patients by comparing the hazard ratio of death at 3 months in patients with and without hyponatraemia. As shown in table 5, the presence of hyponatraemia was associated with an increased risk of death in all subpopulations of patients evaluated: patients with ascites, hepatic encephalopathy, gastrointestinal bleeding, spontaneous bacterial peritonitis or renal failure. Serum sodium was an independent predictor of survival in patients with low and high MELD scores $(<21$ and $\geqslant 21$, respectively).

\section{DISCUSSION}

The current study reports the results of the analysis of survival and prognostic factors of a large cohort of patients with cirrhosis listed for transplantation in a single institution over a 5 -year period. Several characteristics of this cohort make it unique in the assessment of prognostic factors of survival of patients with cirrhosis awaiting liver transplantation. First, it includes a relatively large number of patients treated in a single

Table 3 Regression coefficients, odds ratios and $95 \% \mathrm{Cl}$ of odds ratios of variables with independent predictive value of survival at 3 and 12 months*

\begin{tabular}{lrl}
\hline & Coefficient & OR $(95 \% \mathrm{Cl})$ \\
\hline 3 months & 0.102 & $1.1(1.07$ to 1.142$)$ \\
$\begin{array}{l}\text { MELD } \\
\text { Serum sodium }\end{array}$ & -0.095 & $0.90(0.87$ to 0.94$)$ \\
$\begin{array}{l}12 \text { months } \\
\text { MELD }\end{array}$ & 0.088 & $1.091(1.05$ to 1.138$)$ \\
$\quad$ Serum sodium & -0.128 & $0.88(0.845$ to 0.916$)$ \\
\hline
\end{tabular}

MELD, model for end-stage liver disease; OR, odds ratio.

*Both models were validated with the bootstrap method. In the bootstrap validations for both models, the same variables entered the bootstrapderived models most frequently. In fact, MELD score and serum sodium concentration appeared in 94 and $100 \%$ of the analyses for 3-month survival, and in 100 and $99 \%$ of the analyses for 12-month survival, respectively. Moreover, estimated models using only MELD score and serum sodium concentration as independent predictors were observed in $76 \%$ at 3 months. At 12 months, the mentioned model was estimated in $38 \%$ of the bootstrapped samples, and in only $1 \%$ of the models these two predictors did not appear simultaneously. institution following standardised protocols for the management of complications of cirrhosis developing during the waiting time. Secondly, the study period was restricted to 5 years, which minimises the impact of changes in patients' management over time. Thirdly, during the period of the study, the major determinant of organ allocation was time on the waiting list. Finally, although this is a retrospective study, all variables evaluated as prognostic factors were collected prospectively in a large database at the time of inclusion of patients on the waiting list.

The results of the current study confirm previous data indicating that the MELD score is an independent predictor of survival in patients with cirrhosis awaiting liver transplantation. ${ }^{29}{ }^{30}$ MELD score at the time of listing was an independent predictor of survival at both 3 and 12 months after inclusion of patients on the waiting list. The fact that the allocation of organs in this cohort of patients was done by time on the waiting list reinforces the value of the MELD score as the best method currently available to allocate organs when a system based on the severity of the liver disease is to be used. Comparison of ROC curves assessing the relationship between MELD score and 3- and 12-month survival probability indicates that the MELD score has a similar prognostic accuracy in the assessment of 3-month survival compared with 12-month survival (c-statistic 0.79 and 0.77 , respectively). Moreover, a close look at the relationship between MELD score and 3month survival probability indicates that a major change in survival probability occurs in values of MELD ranging from 15 to 40 (fig 2). In this range, an increase in one point in the MELD score represents an $8 \%$ decrease in 3-month survival probability. Another interesting finding of the current study was that patients with a MELD score lower than 15 have a very high probability of survival at both 3 and 12 months after listing (96 and $87 \%$, respectively). This survival probability is similar to that reported in most transplant centres after transplantation of patients with cirrhosis. ${ }^{31}{ }^{32}$ Patients with low MELD scores are commonly listed for transplantation in transplant programmes using allocation systems based on time on the waiting list, as was the case in the current study. However, the current data showing very low waiting list mortality in this patient population cast doubts about the convenience of listing patients with low MELD scores in transplant programmes in which the allocation system is based on the severity of liver disease. An interesting and somewhat surprising finding of the current study was that hepatorenal syndrome, although significantly associated with prognosis in 

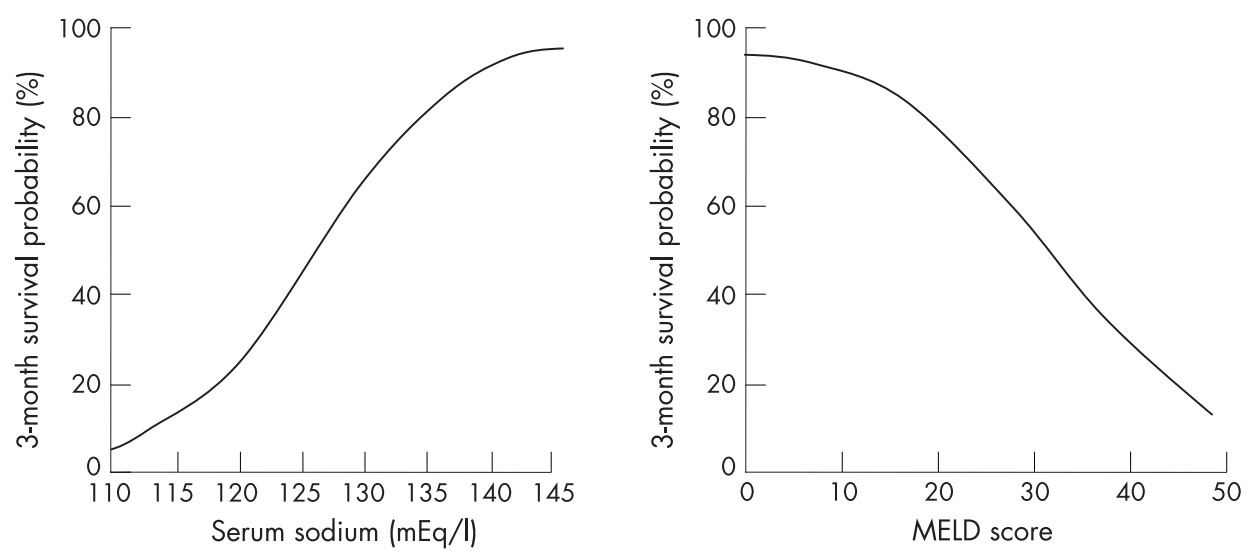

Figure 2 Relationship between serum sodium (left) and model for end-stage liver disease (MELD) score (right) and estimated 3 -month probability of survival in all patients included.

the univariate analysis, was not an independent predictive factor of survival in the multivariable analysis. Although we do not have a complete explanation for this finding, it may be due, at least in part, to the fact that most patients with hepatorenal syndrome were treated with terlipressin and albumin before transplantation, ${ }^{18}$ which could have resulted in an improvement of survival of these patients.

The results of the current study are in keeping with several recent studies in patients with cirrhosis awaiting liver transplantation as well as with previous studies in patients with cirrhosis and ascites indicating that serum sodium concentration is a very good marker of survival, at both 3 and 12 months after inclusion of patients on the waiting list. ${ }^{8-10}$ Serum sodium concentration has also recently been shown to be a good marker of outcome after transplantation. ${ }^{33}$ Comparison of ROC curves assessing the relationship between serum sodium concentration and 3- and 12-month survival probability indicates that serum sodium concentration has better prognostic accuracy in the assessment of 3-month survival than 12-month survival (c-statistic 0.83 and 0.70 , respectively). On the other hand, a close look at the relationship between serum sodium concentration and 3-month survival probability indicates that a major change in survival probability occurs in values of serum sodium ranging from 120 to 135 (fig 2). In this range, a reduction in $1 \mathrm{mEq} / \mathrm{l}$ in serum sodium concentration is associated with a $12 \%$ decrease in 3 -month survival probability. Another relevant clinical finding of this study was that patients without hyponatraemia but with a serum sodium concentration lower than normal values (ie, patients with serum sodium between 130 and $135 \mathrm{mEq} / \mathrm{l}$ ) have a 12-month probability of survival significantly greater than that of patients with hyponatraemia (serum sodium $>130 \mathrm{mEq} / \mathrm{l}$ ) but lower than that of patients with normal serum sodium concentration (fig 3).

The reason why the MELD score is a good marker of prognosis in patients with cirrhosis who are candidates for liver transplantation is probably related to the fact that MELD combines two parameters, bilirubin and INR, that are sensitive markers of liver function, together with serum creatinine, a marker of renal function. ${ }^{2}$ The severity of both liver and renal dysfunction has been shown to correlate with prognosis in patients with cirrhosis. ${ }^{34}{ }^{35}$ In contrast, the explanation for why serum sodium concentration is a good marker of prognosis in patients with cirrhosis is uncertain and has not been specifically investigated. It might be that serum sodium concentration reflects the severity of liver failure, because patients with hyponatraemia commonly have a more advanced liver disease compared with that of patients without hyponatraemia. ${ }^{33}{ }^{36}$ However, the results of this study as well as those from several previous studies ${ }^{8-10}$ indicate that the prognostic value of hyponatraemia is independent of that of the MELD score, which appears to dissociate the predictive value of serum sodium from that of liver and renal failure. Alternatively, it is possible that serum sodium concentration predicts prognosis

\begin{tabular}{|c|c|c|c|c|}
\hline & Dead $(n=64)$ & Alive ( $n=244$ ) & $\mathrm{p}$ Value & Likelihood ratio \\
\hline Age (years) & $52(10)$ & $52(9)$ & 0.9 & \\
\hline Ascites & $60(94 \%)$ & $211(87 \%)$ & & \\
\hline Controlled & $14(23 \%)$ & $98(46 \%)$ & 0.001 & 9.3 \\
\hline Uncontrolled & $46(77 \%)$ & $113(54 \%)$ & & \\
\hline Hepatic encephalopathy & $38(59 \%)$ & $107(44 \%)$ & 0.02 & 4.7 \\
\hline GI bleeding & $20(21 \%)$ & $71(29 \%)$ & 0.42 & 0.1 \\
\hline SBP & $19(30 \%)$ & $64(26 \%)$ & 0.348 & 0.28 \\
\hline Renal failure* & $13(20 \%)$ & $26(11 \%)$ & 0.04 & 3.8 \\
\hline Hepatorenal syndrome $†$ & $9(15 \%)$ & $11(5 \%)$ & 0.01 & 6.38 \\
\hline Bilirubin (mg/dl) & $6.7(7.1)$ & $3.7(3.5)$ & 0.002 & \\
\hline Albumin $(\mathrm{g} / \mathrm{l})$ & $28(5)$ & $31(5)$ & 0.000 & \\
\hline INR & $2(1)$ & $1.6(0.8)$ & 0.03 & \\
\hline Creatinine (mg/dl) & $1.2(1.1)$ & $1.2(1.0)$ & 0.453 & \\
\hline Sodium (mEq/l) & $132(6)$ & $136(4)$ & 0.000 & \\
\hline MELD score & $21(6)$ & $16(5)$ & 0.000 & \\
\hline
\end{tabular}

GI, gastrointestinal; INR, international normalisation ratio; MELD, model for end-stage liver disease; SBP, spontaneous bacterial peritonitis.

${ }^{*}$ As defined by serum creatinine $\geqslant 1.5 \mathrm{mg} / \mathrm{dl}$.

†All patients had type 2 hepatorenal syndrome. 


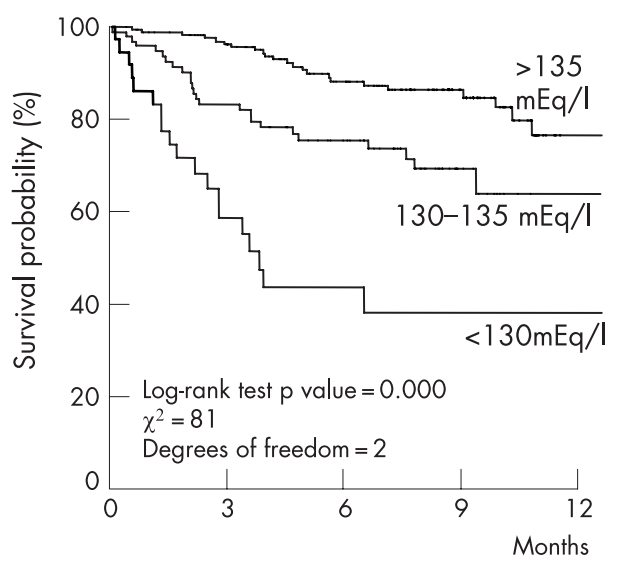

Serum sodium $>135 \mathrm{mEq} / \mathrm{l}(\mathrm{n}=173)$

Serum sodium $131-135 \mathrm{mEq} / \mathrm{l}(\mathrm{n}=98)$ Serum sodium $<130 \mathrm{mEq} / \mathrm{l}(\mathrm{n}=37)$

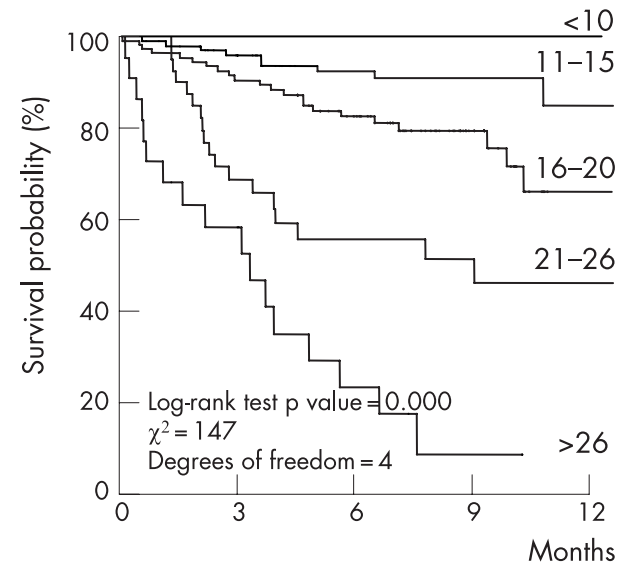

Figure 3 One year-survival before transplantation of the whole population of patients with cirrhosis according to different values of serum sodium (left) and model for end-stage liver disease (MELD) score (right). because it reflects the severity of circulatory failure associated with advanced cirrhosis, as hyponatraemia is due to a nonosmotic hypersecretion of vasopressin, which in turn is dependent on the degree of impairment in circulatory function. ${ }^{21} 22{ }^{37}$ Finally, hyponatraemia has been reported as a predisposing factor for the development of hepatic encephalopathy, ${ }^{38-40}$ although evidence supporting such a relationship is still limited.

Considering the results of previous studies as well as those of the current study, the question arises as to whether serum sodium concentration should be used to improve the prognostic accuracy of the MELD score in view of its simplicity and high predictive value. To assess the possible value of serum sodium in improving the accuracy of the MELD score in the evaluation of prognosis, serum sodium concentration was incorporated in the calculation of the MELD score, using a formula described recently. ${ }^{28}$ A MELD-Na score was then calculated for each patient and its accuracy in predicting prognosis was compared with that of the MELD score and serum sodium using ROC curves. As shown in fig 4, no significant differences were observed between the predictive value of MELD-Na at 3 and 12 months and those of MELD score and serum sodium concentration calculated individually. Discrepancies between the results of the current study and those of previous studies ${ }^{10} 28$ may be related to differences in the patient population, methods used to incorporate sodium in the MELD formula or the method used for organ allocation. On the other hand, it is important to point out that the sample size of the current study was not high enough to rule out completely a better predictive value of MELD-Na compared with MELD or serum sodium alone. In fact, the estimated power of the study was low, $11 \%$ at 3 months and $30 \%$ at 12 months. An estimated power $>80 \%$ would have required the inclusion of 1268 patients which is difficult to achieve in single-centre studies, unless the study period is markedly prolonged, an approach that does not seem appropriate because it will increase the heterogeneity of the patient population. Therefore, multicentre studies with very large sample sizes would be required to test the superiority of MELD-Na with respect to MELD alone in the assessment of prognosis of patients with cirrhosis awaiting liver transplantation.

While awaiting the results of these studies, it is important to ponder the appropriateness of including serum sodium as a new variable in a score for organ allocation in liver transplantation. A

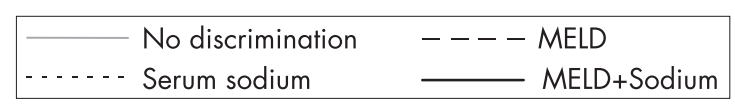

3-month survival

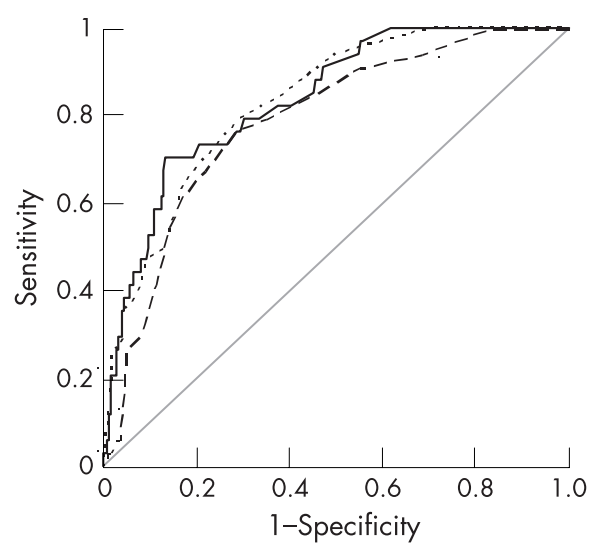

12-month survival

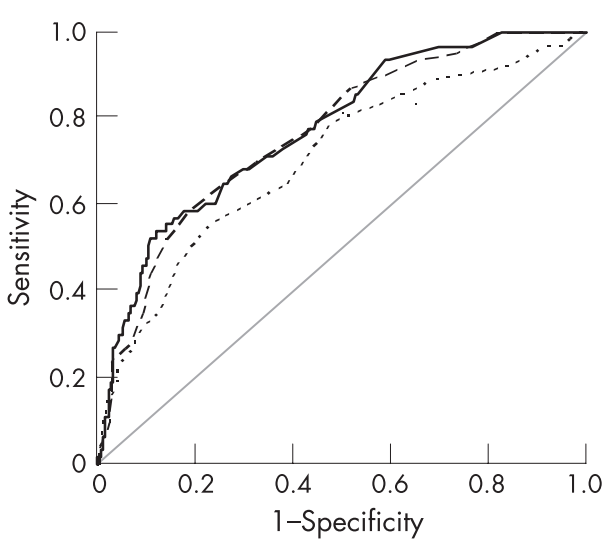

Figure 4 Receiver operating characteristic (ROC) curves of the model for end-stage liver disease (MELD) score (c-statistic (SEM) 0.79 (0.04) at 3 months and $0.77(0.03)$ at 12 months), serum sodium concentration (cstatistic (SEM) $0.82(0.03)$ and 0.70 (0.04) at 3 and 12 months, respectively) and MELD plus serum sodium concentration (c-statistic (SEM) $0.83(0.03)$ and $0.77(0.03)$, at 3 and 12 months, respectively), and survival probability at 3 (left) and 12 months (right). MELD plus serum sodium concentration was calculated as described in Hosmer and Lemeshow. ${ }^{23} \mathrm{p}=$ not significant for all comparisons. 
Table 5 Mortality risk expressed by hazard ratio in patients included in the study with or without hyponatraemia categorised according to the presence of major complications of cirrhosis

\begin{tabular}{lll}
\hline & Hyponatraemia* & No hyponatraemia \\
\hline Ascites & $\mathrm{n}=37$ & $\mathrm{n}=234$ \\
& $5.9(2.9$ to 11.7$)$ & 1.0 (reference) \\
Hepatic encephalopathy & $\mathrm{n}=20$ & $\mathrm{n}=125$ \\
& $7.8(3.1$ to 19.8$)$ & 1.0 (reference) \\
Variceal bleeding & $\mathrm{n}=9$ & $\mathrm{n}=82$ \\
& $11.5(3.3$ to 40$)$ & 1.0 (reference) \\
Spontaneous bacterial & $\mathrm{n}=15$ & $\mathrm{n}=68$ \\
peritonitis & $13.6(4.0$ to 45$)$ & 1.0 (reference) \\
Renal failure & $\mathrm{n}=11$ & $\mathrm{n}=28$ \\
& $16.5(1.9$ to 142$)$ & 1.0 (reference) \\
\hline
\end{tabular}

Data are relative risk $(95 \% \mathrm{Cl})$

*Hyponatraemia was defined as serum sodium $<130 \mathrm{mEq} / \mathrm{l}$.

new variable should ideally meet the following criteria: good correlation with survival; easy measurement; wide availability; and stability. While serum sodium meets the first three criteria, it does not meet the fourth. In fact, serum sodium levels may have important fluctuations after simple therapeutic manoeuvres, which do not necessarily reflect changes in the severity of the disease. For example, administration of diuretics is commonly associated with marked changes in serum sodium concentration, which are of $4 \mathrm{mEq} / \mathrm{l}$ on average and can be as high as 10$15 \mathrm{mEq} / \mathrm{l}$, and are often reversible after diuretic withdrawal. ${ }^{42}$ Moreover, serum sodium may decrease markedly after administration of oral or intravenous hypotonic fluids due to the impaired capacity to eliminate solute-free water commonly present in patients with advanced cirrhosis. ${ }^{43}$ Finally, the serum sodium concentration increases markedly after the administration of drugs that antagonise selectively the V2 receptors of the antidiuretic hormone. Phase 2 studies have shown that these drugs increase serum sodium concentration and improve the management of ascites in patients with cirrhosis and hyponatraemia, and may be available soon for use in clinical practice. ${ }^{44-46}$ If the beneficial effects of these drugs are confirmed in phase 3 studies, which are currently underway, the addition of serum sodium as a variable in a new score to be used for organ allocation may be an obstacle for the use of these drugs in patients awaiting liver transplantation.

In conclusion, the results of the current study indicate that the MELD score and serum sodium concentration are the only independent predictive factors of 3- and 12-month survival in patients with cirrhosis awaiting liver transplantation. Serum sodium concentration is equally accurate in the assessment of prognosis in different subpopulations of patients with cirrhosis categorised according to the major complication developed before listing in our patient population. The addition of serum sodium to the MELD score does not appear to improve significantly the prognostic accuracy of the MELD score alone. Nevertheless, additional studies in large patient populations should be performed to address this issue further.

\section{ACKNOWLEDGEMENTS}

The authors thank Dr Juan Gonzalez-Abraldes for his help in the evaluation of data.

\footnotetext{
Authors' affiliations

Maria-Carlota Londoño, Andrés Cárdenas, Mónica Guevara, Dara de las Heras, Miguel Navasa, Antoni Rimola, Vicente Arroyo, Pere Ginès, Liver Unit, Hospital Clínic, Universitat de Barcelona, Institut de Investigacions Biomèdiques August Pi-Sunyer (IDIBAPS), CIBER de Enfermedades Hepáticas y Digestivas (CIBEREHD) and Centro de Investigaciones Biomédicas Esther Koplowitz (CIBEK), Barcelona, Spain
}

Juan-Carlos Garcia-Valdecasas, Department of Surgery, Hospital Clínic, Universitat de Barcelona, Institut de Investigacions Biomèdiques August $\mathrm{Pi}$ Sunyer (IDIBAPS), CIBER de Enfermedades Hepáticas y Digestivas (CIBEREHD) and Centro de Investigaciones Biomédicas Esther Koplowitz (CIBEK), Barcelona, Spain

Llorenç Quintó, Department of Biostatistics, Hospital Clínic, Universitat de Barcelona, Institut de Investigacions Biomèdiques August Pi-Sunyer (IDIBAPS), CIBER de Enfermedades Hepáticas y Digestivas (CIBEREHD) and Centro de Investigaciones Biomédicas Esther Koplowitz (CIBEK), Barcelona, Spain

This study was supported by grants from Fondo de Investigación Sanitaria (FIS 05/0246) and Ministerio de Educación y Ciencia (SAF 2005-01917). Mónica Guevara was supported by a grant from Fondo de Investigación Sanitaria (FISO1/3045). Maria-Carloto Londoño has received financial support from the Fundacion Banco Bilbao Vizcaya Argentaria.

Competing interests: None.

\section{REFERENCES}

1 Kamath PS, Wiesner RH, Malinchoc $M$, et al. A model to predict survival in patients with end-stage liver disease. Hepatology 2001;33:464-70.

2 Wiesner RH, McDiarmid SV, Kamath PS, et al. MELD and PELD: application on survival models to liver allocation. Liver Transp/ 2001;7:567-80.

3 Freeman RB Jr, Wiesner RH, Harper A, et al. UNOS/OPTN Liver Disease Severity Score, UNOS/OPTN Liver and Intestine, and UNOS/OPTN Pediatric Transplantation Committees. The new liver allocation system: moving toward evidence-based transplantation policy, Liver Transp/ 2002;8:851-8.

4 Everson GT. MELD: the answer or just more questions? Gastroenterology 2003; 124:251-4.

5 Freeman RB. MELD: the holy grail of organ allocation? J Hepatol 2004;42:16-20.

6 Cholongitas E, Senzolo M, Triantos C, et al. MELD is not enough-enough of MELD? J Hepatol 2005:42:475-7.

7 Durand F, Valla D. Assessment of the prognosis of cirrhosis: Child-Pugh versus MELD. J Hepatol 2005;42:S100-7.

8 Heuman DM, Abou-Assi SG, Habib A, et al. Persistent ascites and low serum sodium identify patients with cirrhosis and low MELD scores who are at high risk for early death. Hepatology 2004;40:802-10.

9 Biggins SW, Rodriguez HJ, Bacchetti P, et al. Serum sodium predicts mortality in patients listed for liver transplantation. Hepatology 2005;41:32-9.

10 Ruf AE, Kremers WK, Chavez LL, et al. Addition of serum sodium into the MELD score predicts waiting list mortality better than MELD alone. Liver Transpl 2005;11:336-43.

11 Bustamante J, Rimola A, Ventura PJ, et al. Prognostic significance of hepatic encephalopathy in patients with cirrhosis. J Hepatol 1999;30:890-5.

12 Davis CL. Impact of pretransplant renal failure: when is listing for kidney-liver indicated? Liver Transpl 2005; 11:S35-44.

13 Moore KP, Wong F, Gines P, et al. The management of ascites in cirrhosis: report on the consensus conference of the International Ascites Club. Hepatology 2003:38:258-66.

14 Ginès $P$, Cárdenas A, Arroyo V, et al. Management of cirrhosis and ascites. N Engl J Med 2004;350:1646-54.

15 Pugh RN, Murray-Lyon IM, Dawson JL, et al. Transection of the oesophagus for bleeding oesophageal varices. Br J Surg 1973;60:646-9.

16 Cholongitas E, Senzolo M, Pathc D, et al. Review article: scoring systems for assessing prognosis in critically ill adult cirrhotics. Aliment Pharmacol Ther 2006;24:453-64.

17 Cárdenas A, Ginès P. Management of complications of cirrhosis in patients awaiting liver transplantation. J Hepatol 2005;42(Suppl 1):S124-33.

18 Ginès $\mathbf{P}$, Guevara $M$, Arroyo $V$, et al. Hepatorenal syndrome. Lancet 2003;362:1819-27

19 Bosch J, Abraldes JG, Groszmann R. Current management of portal hypertension. J Hepatol 2003;38:S54-68.

20 de Franchis R. Evolving consensus in portal hypertension. Report of the Baveno IV consensus workshop on methodology of diagnosis and therapy in portal hypertension. J Hepatol 2005;43:167-76.

21 Ginès $\mathbf{P}$, Berl T, Bernardi $M$, et al. Hyponatremia in cirrhosis: from pathogenesis to treatment. Hepatology 1998;28:851-64.

22 Arroyo V, Ginès P, Gerbes AL, et al. Definition and diagnostic criteria of refractory ascites and hepatorenal syndrome in cirrhosis. International Ascites Club. Hepatology 1996;23:164-76.

23 Hosmer DW, Lemeshow S. Applied logistic regression. New York: Wiley, 1989

24 Collett D. Modelling binary data. London: Chapman \& Hall, 1991.

25 Balckstone EH. Breaking down barriers: helpful breakthrough statistical methods you need to understand better. J Thorac Cardiovasc Surg 2001;122:430-9.

26 StataCorp. Stata Statistical Softwware: Release 9. College Station, TX: StataCorp P, 2005.

27 Hanley JA, McNeil BJ. The meaning and use of area under a receiver operating characteristic (ROC) curve. Radiology 1982;143:29-36.

28 Biggins SW, Kim WR, Terrault NA, et al. Evidence-based incorporation of serum sodium concentration into MELD. Gastroenterology 2006;130:1652-60.

29 Wiesner R, Edwards E, Freeman R, et al. United Network for Organ Sharing Liver Disease Severity Score Committee. Model for end-stage liver disease (MELD) and allocation of donor livers. Gastroenterology 2003;124:91-6. 
30 Botta F, Giannini E, Romagnoli P, et al. MELD scoring system is useful for predicting prognosis in patients with liver cirrhosis and is correlated with residual liver function: a European study. Gut 2003;52:134-9.

31 Kim WR, Therneau TM, Benson JT, et al. Deaths on the liver transplant waiting list: an analysis of competing risks. Hepatology 2006;43:345-51.

32 Roberts MS, Angus DC, Bryce CL, et al. Survival after liver transplantation in the United States: a disease-specific analysis of the UNOS database. Liver Transpl 2004; 10:886-97.

33 Londoño MC, Guevara M, Rimola A, et al. Hyponatremia impairs early posttransplantation outcome in patients with cirrhosis undergoing liver transplantation. Gastroenterology 2006;130:1135-43.

34 Llach J, Ginès P, Arroyo V, et al. Prognostic value of arterial pressure, endogenous vasoactive systems, and renal function in cirrhotic patients admitted to the hospital for the treatment of ascites. Gastroenterology 1988;94:482-7.

35 Fernández- Esparrach G, Sánchez-Fueyo A, Ginès $P$, et al. A prognostic model for predicting survival in cirrhosis with ascites. J Hepatol 2001;34:46-52.

36 Borroni G, Maggi A, Sangiovanni A, et al. Clinical relevance of hyponatraemia for the hospital outcome of cirrhotic patients. Dig Liver Dis 2000;32:605-10.

37 Ishikawa S, Schrier RW. Pathogenesis of hyponatremia: the role of arginine vasopressin. In: Ginès P, Arroyo V, Rodés J, Schrier RW, eds. Ascites and renal dysfunction in liver disease. Oxford: Blackwell Publishing, 2005, 305-14.

38 Baccaro ME, Guevara M, Torre A, et al. Hyponatremia predispones to hepatic encephalopathy in patients with cirrosis. Results of prospective study with timedependent analysis [abstract]. Hepatology. 2006;44: 233 A).
39 Cordoba J, Gottstein J, Blei AT. Chronic hyponatremia exacerbates ammoniainduced brain edema in rats after portacaval anastomosis. $J$ Hepatol 1998;29:589-94.

40 Haussinger D, Kircheis G, Fischer R, et al. Hepatic encephalopathy in chronic liver disease: a clinical manifestation of astrocyte swelling and low-grade cerebral edema? J Hepatol 2000;32:1035-8.

41 Jalan R, Elton RA, Redhead DN, et al. Analysis of prognostic variables in the prediction of mortality, shunt failure, variceal rebleeding and encephalopathy following the transjugular intrahepatic portosystemic stent-shunt for variceal haemorrhage. J Hepatol 1995;23:123-8.

42 Ginès $P$, Arroyo V, Quintero $E$, et al. Comparison of paracentesis and diuretics in the treatment of cirrhotics with tense ascites. Results of a randomized study. Gastroenterology 1987;93:234-41.

43 Arroyo V, Rodés J, Gutierrez-Lizarriaga MA, et al. Prognostic value of spontaneuous hyponatremia in cirrhosis with ascites. Am J Dig Dis 1976;21:249-56.

44 Ginès $P$, Wong $F$, Milutinovic $S$, et al. Effects of satavaptan (SR121463B), a selective vasopressin $\mathrm{V} 2$ receptor antagonist, on serum sodium concentration and ascities in patients with cirrhosis and hyponatremia. J Hepatol 2006;44:S270.

45 Wong F, Ginès $\mathrm{P}$, Watson $\mathrm{H}$, et al. Effects of a selective vasopressin V 2 receptor antagonist, Satavaptan (SR121463B), on recurrence of ascites after large volume paracentesis [abstract]. Hepatology. 2006;44: 256 A).

46 Schrier RW, Gross P, Gheorghiade $M$, et al. Tolvaptan, a selective oral vasopressin $\mathrm{V}_{2}$-receptor antagonist, for hyponatremia. $N$ Engl J Med 2006;355:2099-1 12.

\section{Access the latest content chosen by our Editors}

BMJ Journals editors select an article from each issue to be made free online immediately on publication. Other material is free after 12 months to non-subscribers. Access the Editor's Choice from the home page - or expand your horizons and see what the other BMJ Journals editors have chosen by following the links on any BMJ Journal home page. 